\title{
Effects of semantic and cheremic context on acquisition of manual signs
}

\author{
CAROL BERGFELD MILLS \\ University of Maryland, College Park, Maryland 20742 \\ and \\ LINDA J. WELDON \\ Advanced Research Resources Organization, Bethesda, Maryland 20814
}

In two experiments, sign-naive subjects acquired the meanings for manual signs of American Sign Language by learning to respond with the English word equivalents when signs were presented. The results showed that when the signs on a to-be-learned list were related to each other in handshape configuration (cheremically similar), they were more difficult to acquire than when semantically similar. Whether the similar signs were grouped together during presentation or were separated by other dissimilar signs had no effect on the number of signs correctly acquired. These results were the same for the identical signs learned in the cheremically or semantically similar contexts as for the lists as a whole. The results have implications for teaching sign language to hearing adults.

The purpose of the present experiments was to answer two questions concerning the ability of hearing adults to acquire manual signs (e.g., signs of American Sign Language): (1) Are signs correctly acquired after fewer trials when they are presented in the context of signs that look similar (cheremic similarity) or in the context of signs that are similar in meaning (semantic similarity)? (2) Are signs correctly acquired after fewer trials when similar signs are grouped in a given learning session or when similar signs are not grouped together?

Two skills are important in acquiring a language: receptive skills (recognizing or knowing what is said) and productive skills (being able to say something). The present studies concern the acquisition of the receptive skills, that is, the ability to "recognize" manual signs by responding with English word equivalents when the signs are presented.

The recognition of manual signs may be thought of as a paired associate task in which the visual presentation of the sign is the stimulus and an English word corresponding to a meaning of the sign is the response. A well established phenomenon in paired associate learning is that similarity among items retards acquisition

Experiment 1 was conducted when the first author held a postdoctoral fellowship at Gallaudet College. Computer services were provided by the Computer Science Center of the University of Maryland. The authors are grateful to Nancy S. Anderson, Charles Clifton, Judson Mills, Horace Reynolds, and an anonymous reviewer for comments on this manuscript. Jessica Barnes and Christine Kispert aided in the analysis of the difficulty ratings. Portions of this research were presented at the meeting of the Psychonomic Society, St. Louis, Missouri, November 1980. Send reprint requests to Carol Bergfeld Mills, Psychology Department, University of Maryland, College Park, Maryland 20742. (e.g., Goss \& Nodine, 1965; Kintsch, 1977). Increasing semantic similarity of items in a paired associate list produces interference in learning (e.g., Runquist, 1968; Underwood, 1953a), as does increasing formal similarity (e.g., Nelson \& Rowe, 1969; Underwood, 1953b). Semantic similarity for a written or oral language is the same as for sign language: similarity based on meaning. Graphemic similarity for a written language and phonological similarity for oral language (formal similarity) seem to be analogous to cheremic similarity for sign language. Formal characteristics of American Sign Language were first discussed by Stokoe (1960). Stokoe's analysis suggested that signs require at least three simultaneous attributes: (1) the configuration of the hand or hands in making the sign, (2) the location of the sign in relation to the signer's body, and (3) the movement of the hand or hands. Each of these three attributes has a number of values or cheremes. Based on Stokoe's analysis, signs that are cheremically similar would have one or two cheremes that are the same.

To reduce the interference caused by either formal or semantic similarity, it seems that the learner needs to clearly differentiate between items (Anderson \& Bower, 1973; Battig, 1968; Gibson, 1940). Differentiation appears to involve the selection or utilization of a unique characteristic or cluster of characteristics to facilitate encoding and serve as retrieval cues. The overall difficulty of a list would be determined by the ease with which unique characteristics can be located. The ability to differentiate between items may depend to some extent on the learners' previous knowledge. Differentiation may be more difficult on a dimension that is unfamiliar to learners than on a dimension that is very familiar (cf. Stein, 1978). 
For signs, differentiation among similar physical features should be more difficult than differentiation among semantic features. There seem to be fewer unique characteristics that can be used to differentiate among cheremically similar signs than among semantically similar signs. As a result, the deeper levels of processing or encoding for the cheremically similar signs may be limited by the ability to differentiate, whereas this would not seem to be the case for semantically similar signs. In addition, adult sign learners, who are learning sign language as a second language, would have more difficulty in determining what the important unique attributes are for the cheremically similar signs than for semantically similar signs. Based on previous experience, these sign learners have an appropriate schema for dealing with semantic similarities and therefore can precisely encode information on that dimension of similarity. However, adult sign learners who are naive with respect to sign language do not have an appropriate schema for dealing with the attributes of handshape, movement, and location. The lack of a schema would make cheremically similar signs difficult to encode.

The second question addressed by the present study is whether it is easier to recognize signs when similar signs are presented together in a given list or when similar signs are not grouped together. Assuming that the main difficulty would be in differentiating the physical characteristics, then grouping similar signs should facilitate encoding for signs that are cheremically similar, because it is easier to determine what the unique physical characteristics of the related signs are when they are presented successively. On the other hand, for semantically similar signs, there should be no particular advantage in differentiating between signs when they are in groups (compared with when similar signs are not grouped together). For semantically similar signs (for which there are minimum cheremic relationships), there are many unique physical characteristics that can be used to differentiate between a given sign and other signs.

Previous paired associate studies provide some support for these predictions. Studies with formally similar stimuli (Gagné, 1950; Rotberg \& Woolman, 1963) showed better learning performance when similar stimuli were grouped than when similar stimuli were not grouped, whereas studies with semantically similar stimuli (Runquist \& Runquist, 1978) showed no difference in learning rate. However, the results of other studies have not been consistent with the present predictions (e.g., Keister, 1972; Rothkopf, 1958).

The results of the present experiments may have practical implications for how to facilitate sign recognition in hearing adult learners. Sign language text writers generally do not agree on how signs should be introduced to facilitate learning. Some writers, such as T. J. O'Rourke in A Basic Course in Manual Communication (1973), tend to introduce cheremically similar signs in a given lesson. When this text is used for a course, signs that are cheremically similar tend to be presented in the same class period. Thus, the cheremically similar signs are presented grouped together. Other text writers use different methods for introducing signs, such as L. Riekehof in The Joy of Signing (1978). Riekehof tends to introduce signs that are semantically similar in a given lesson. Thus, when this text is used for a course, signs that are semantically similar are presented in a given class period and the similar signs are presented grouped together. This type of organization usually results in the cheremically similar signs not being grouped together. The present research may provide information about which type of sign language textbook facilitates sign recognition for hearing adults.

Because of an interest in practical implications, the subjects' task was made as much like the task of actual sign language learners as possible, while still maintaining strict experimental procedures. Real signs from American Sign Language, rather than created signs, were used, and an appropriate English word, rather than nonsense materials, inappropriate responses, or digits, was paired with each sign. As a consequence of attempting to maximize the applicability of the results, another factor covaried with semantic and cheremic similarity. In the semantically similar condition, the English word equivalents (responses) appear to be similar, and in the cheremically similar condition, the signs (stimuli) appear to be similar. Any differences between the semantically similar condition and the cheremically similar condition could be due to differences in the locus of similarity (cf. Goss \& Nodine, 1965).

The covariation of the locus of similarity and semantic vs. cheremic similarity was not considered a problem in the present experiments for two reasons. First, previous research has shown that the effects of similarity are equivalent, regardless of whether the similarity is in the stimulus items or in the response items. Baddeley (1970) found that the degree of impairment in paired associate recall was the same regardless of whether similarity was in the stimulus items, the response items, or both. Horowitz (1962) found equivalent effects of stimulus and response similarity when response learning was minimized. In the present experiments, response learning was expected to be minimal since the responses were common words.

Second, the present experiments were designed to represent the procedures used in the teaching of manual signs. As mentioned previously, usually either semantically similar signs or cheremically similar signs are presented in a given lesson. Since in the typical sign language learning situation, semantic vs. cheremic similarity covaries with the locus of similarity, the covariation of the two factors in the procedure of the present research does not detract from its applied value.

\section{EXPERIMENT 1}

\section{Method}

Experimental design. The subjects' task was to learn the English language equivalents for visually presented signs in five study-test trials. Signs on a given list were either semantically similar (semantic list) or formally similar (cheremic list). The signs on each list were presented so that the similar signs were 
either grouped together (grouped presentation) or not grouped together (scrambled presentation) on the study trials. The design was a 2 by 2 by 5 design with type of list (semantic vs. cheremic) and presentation order (grouped vs. scrambled) as betweensubjects factors and trial (1 to 5) as a within-subjects factor.

Sign selection. There were two lists of 28 signs each, a semantic list and a cheremic list. Each list consisted of eight sets of related signs. There were two sets of Size 2, Size 3, Size 4, and Size 5. The signs for the semantic list were selected so that the signs within a set were related semantically (e.g., were categorically or associatively related), and the signs on the list were relatively unrelated cheremically. One semantic set included signs for foods: potato, milk, pepper, bread, and egg. Another semantic set included signs for associatively related concepts, right and wrong. The signs for the cheremic list were selected so that two of three possible sign attributes (handshape, body location, and movement) were the same for signs within a set (e.g., Stokoe, 1960; Stokoe, Casterline, \& Croneberg, 1965), ${ }^{1}$ and the signs on the list were relatively unrelated semantically. One cheremic set included signs with the same attributes of handshape and location: weight, short, name, and egg. Another cheremic set had signs with the same attributes of handshape and movement: ugly, summer, and dull. The two lists of signs were selected so that the frequency of the English word equivalents for the semantic list was not higher than for the cheremic list. This was done to ensure that better recognition on the semantic list could not be attributed to the frequency of occurrence, since it has been shown that paired associate learning improves with an increase in frequency of the response (cf. Postman, 1962). Based on the KuXera and Francis (1967) word count, the mean frequency for the semantic list was 295.29 and that for the cheremic list was 546.43. Two of the signs on the semantic and cheremic lists were the same, penny and egg (overlapping signs).

There are a limited number of cheremes or values for each sign language attribute (handshape, movement, location). As a result, it was not possible to eliminate completely all cheremic similarity from the semantic list. Likewise, there may be similar semantic characteristics among some items on the cheremic list. "Pure" lists may only be feasible if artificial materials are created.

Presentation order. Each of the two lists of signs, semantic and cheremic, was presented in two different ways, grouped or scrambled. In the grouped presentation, the sets of signs, which were either semantically or cheremically similar, were presented successively during the study trials. The order of presentation of the sets, as well as the order of the signs within each set, was a different random order for each of five study trials. For the semantic-grouped condition, one study trial might start with sets of Size 2-4-3, such as right, wrong; France, England, America, Spain; money, penny dollar. Another study trial might start with sets of Size 4-2-2, such as America, England, Spain, France; wrong, right; day, night.

For the scrambled presentation, eight sets of signs were also used with two sets each of Size 2, Size 3, Size 4, and Size 5. However, the sets were formed by randomly assigning signs to the eight sets, with the constraint that no related signs occurred within a given set. Thus, the scrambled presentation resulted in the same signs always being presented in sets (but not in the same order), as was also true in the grouped presentation, but the signs within a given set for the scrambled presentation were not semantically or cheremically related. This type of scrambled presentation was chosen in order to make the scrambled and grouped conditions more comparable.

The same five orders were used for the scrambled study trials as for the grouped study trials by yoking the scrambled sets (and the signs within those sets) to grouped sets of the same size. For the test trials, signs were not arranged by set but were five com pletely random orders. The same test trial orders were used for grouped and scrambled conditions.
Stimulus materials. A videotape was made of a female signer making the signs in the appropriate order for the study and test trials. The signer used very little facial expression or body movement in order that cues other than hand movements could not be used as cues for sign recognition. During the study trials, the signer pronounced the English word equivalent corresponding to the sign. The signs were produced with very careful movements at the rate of approximately one sign every $3 \mathrm{sec}$, with no indication of the beginnings or endings of sets. During the test trials, the signer produced the signs without pronouncing the English word equivalent at the rate of approximately one sign every $8 \mathrm{sec}$. Two seconds prior to making a sign, she gave the stimulus number of the sign to help subjects keep track of the number and to warn subjects that a sign was coming. Between each sign on both the study and test trials, the signer returned to a neutral hand position.

After recording each study trial, two interconnected recorders were used to simultaneously record the test trials on both the grouped and scrambled tapes for a given list (semantic or cheremic). This was done to ensure that differences in sign clarity on the test trials could not contribute to the grouped vs. scrambled effect.

Procedure. Subjects were tested in groups of 2 to 10 individuals. One of the four conditions was randomly assigned for each group of subjects, with the restriction that an equal number of subjects participated in each of the four conditions. Subjects were seated in a small classroom with low illumination. A 19 in. black-and-white video monitor, in full view for all subjects, was used to view the experimental materials.

Subjects were told that they were going to see a videotape of signs and that they should try to remember the English word for each sign that they saw. All subjects were informed that some signs were similar to each other in meaning and some signs looked similar. They were instructed that there would be five presentations of the list of signs and that after each presentation, a test trial would occur. They were also informed that during the test trials, the signer would make the sign, and they were going to be asked to write down the English word for the sign on a numbered sheet of paper.

In scoring the response, homonyms of the English words were accepted as correct because the words were presented auditorily.

Subjects. The subjects were 80 students from psychology classes at the University of Maryland who participated in order to gain extra course credit. Three additional subjects were tested but their data were eliminated because they reported prior sign and/or fingerspelling knowledge.

\section{Results}

The percentage of correct responses per test trial for each of the four conditions is shown in Table 1. An analysis of variance was performed on the mean number of correct responses with semantic vs. cheremic, grouped

Table 1

Mean Percentage Correct Responses as a Function of Trial and Condition in Experiment 1

\begin{tabular}{lcccccc} 
& \multicolumn{6}{c}{ Trial } \\
\cline { 2 - 6 } \multicolumn{1}{c}{ Condition } & 1 & 2 & 3 & 4 & 5 & Mean \\
\hline Semantic Scrambled & 48.9 & 81.3 & 92.9 & 96.8 & 97.9 & 83.5 \\
Semantic Grouped & 59.6 & 83.8 & 94.5 & 97.1 & 99.8 & 87.0 \\
Cheremic Scrambled & 27.0 & 56.3 & 74.8 & 85.0 & 89.1 & 66.4 \\
Cheremic Grouped & 26.8 & 57.1 & 75.7 & 84.3 & 89.5 & 66.7 \\
Mean & 40.6 & 69.6 & 84.5 & 90.8 & 94.0 & \\
\hline
\end{tabular}


vs. scrambled, and trial (1-5) as factors. This analysis revealed a significant effect of semantic vs. cheremic $[\mathrm{F}(1,76)=50.13, \mathrm{p}<.001, \mathrm{MSe}=54.67]$ and trial $[F(4,304)=544.64, p<.001, \mathrm{MSe}=5.51]$. There was also a significant interaction between semantic vs. cheremic and trial $[\mathrm{F}(4,304)=17.85, \mathrm{p}<.001$, MSe $=$ 5.51]. The effect of grouped vs. scrambled and the other interactions were not significant. The mean number of correct recognitions per trial was greater for the semantic list (23.87) than for the cheremic list (18.64). Not surprisingly, recognition of the signs improved between Trial 1 and Trial 5, as can be seen in Table 1. A Tukey HSD test showed that for each of the five trials, recognition was significantly better for the semantic list than for the cheremic list $(p<.01)$. As can be seen from Table 1, the interaction between semantic vs. cheremic and trial was probably due to a ceiling effect in the fourth and fifth trials on the semantic list.

The percentage of correct recognitions was analyzed separately for the two overlapping signs. The pattern of results was identical to that for the lists as a whole, with the same significant effects.

To determine which signs were the hardest to differentiate, signs inside the same set or signs outside the set, an analysis of substitution errors (excluding omissions and intrusions) was conducted in a manner similar to that done by other experimenters (e.g., Gagné, 1950; Rotberg \& Woolman, 1963). An inside error was defined as the use of a response word appropriate to a sign from the same set as the sign stimulus, for example, the response "penny" for "dollar" on the semantic list. An outside error was defined as the use of an English equivalent appropriate to a sign in another set on the list, for example, the response "potato" for "dollar" on the semantic list.

The mean number of substitution errors for each condition is presented in Table 2 . The analysis of variance included inside vs. outside errors, semantic vs. cheremic, and grouped vs. scrambled as factors. Prior to this analysis, a logarithmic transformation, $X^{\prime}=\log (X+1)$, was used to achieve homogeneity of variance $\left[F_{\max }(8,20)=\right.$ $2.97, \mathrm{p}>.05]$. The analysis of variance showed the following significant effects: semantic vs. cheremic $[F(1,76)=78.27, p<.001, \mathrm{MSe}=.11] ;$ Semantic vs.

Table 2

Mean Number of Substitution Errors in Experiment 1 for Each Condition for All Trials Combined

\begin{tabular}{clccr}
\hline & \multicolumn{3}{c}{ Presentation Order } \\
List & Error & Grouped & Scrambled & Total \\
\hline \multirow{2}{*}{ Semantic } & Inside & 1.15 & 1.30 & 2.45 \\
& Outside & 3.80 & 2.10 & 5.90 \\
Cheremic & Inside & 9.45 & 12.15 & 21.60 \\
& Outside & 5.30 & 4.35 & 9.65 \\
Combined & Inside & 10.60 & 13.45 & 24.05 \\
& Outside & 9.10 & 6.45 & 15.55 \\
\hline
\end{tabular}

Cheremic by Inside vs. Outside interaction $[F(1,76)=$ $62.72, \mathrm{p}<.001, \mathrm{MSe}=.05] ;$ and Grouped vs. Scrambled by Inside vs. Outside interaction $[F(1,76)=5.77$, $\mathrm{p}<.05, \mathrm{MSe}=.05]$. None of the other main effects or interactions were significant. This analysis showed that more substitution errors were made for the cheremic list (31.25) than for the semantic list (8.35). A Tukey HSD test of the interaction between semantic vs. cheremic and inside vs. outside errors showed that all of the differences between the means were significant $(p<.01)$. More outside errors than inside errors were made on the semantic list (5.90 vs. 2.45 ), but more inside errors than outside errors were made on the cheremic list (21.60 vs. 9.65). More inside and outside errors were made on the cheremic than on the semantic list. The Tukey HSD for the interaction between grouped vs. scrambled and inside vs. outside showed that for the scrambled presentation only, more inside errors than outside errors were made $(13.45$ vs. $6.45, \mathrm{p}<.05)$. It should be noted that this error analysis was based on the actual number of inside and outside errors made and did not take into account the greater probability of the chance occurrence of outside errors.

To summarize, the results of this experiment showed that, as expected on the basis of stimulus differentiation, signs on a list of semantically similar signs (semantic list) were easier to recognize than those on a list of signs related in handshape configuration, location, and/or movement (cheremic list). However, whether the signs that were similar to each other were presented so that the similar signs were grouped together (grouped) or not (scrambled) did not affect overall recognition performance. This pattern of results occurred for the lists as a whole as well as for the overlapping signs. The analysis of substitution errors indicated that more errors were made on the cheremic list than on the semantic list. On the cheremic list, more outside-set errors and particularly more inside-set errors were made than on the semantic list. Unlike the recognition performance, the substitution errors were affected by the grouped vs. scrambled presentation. Scrambled presentation resulted in more substitutions from inside the set than from outside the set.

\section{EXPERIMENT 2}

Experiment 2 employed new lists of signs in an attempt to eliminate an alternative interpretation for the semantic vs. cheremic effect. It might be thought that the individual signs on the semantic list were easier in some respects than those on the cheremic list, even though there was nothing in the sign selection procedure that should have made this so. Replicating the results of Experiment 1 with new lists of signs would make that interpretation unlikely. Experiment 2 also used lists that were more than twice as long as those used in Experiment 1 in an attempt to eliminate a ceiling effect in the 
semantic condition. In addition, the number of overlaping signs was increased in Experiment 2 in order to have a better measure of the effect of semantic and cheremic context on sign recognition.

\section{Method}

The design and procedure were identical to those in Experiment 1 . Two lists of signs, 60 signs for the semantic list and 60 signs for the cheremic list, were selected. Each list consisted of ten sets: four sets of Size 5, four sets of Size 6, one set of Size 7, and one set of Size 9. An example of a semantic set was one that included signs for countries: Holland, France, America, Spain, England, Scotland, and Germany. The signs for the cheremic list were selected so that the signs in a given set had at least one attribute in common. For example, one of the cheremic sets included signs with a similar attribute for handshape and the same attribute for location: gray, football, America, meeting, and machine. For Experiment 2, there were 26 overlapping signs. The lists were selected so that the overlapping signs that occurred in a given set in the semantic list were not in any given set on the cheremic list. Because of the high proportion of overlapping signs in Experiment 2, by necessity some semantically related signs occurred on the cheremic list and vice versa. As in Experiment 1, the frequency of the English word equivalents for the semantic list was not higher than that for the cheremic list. Based on the Kucrera and Francis (1967) word count, the mean frequency for the semantic list was 145.15 and that for the cheremic list was 246.85. In all other respects, the criteria for selecting signs were identical to those used in Experiment 1.

The subjects were run in groups of 10 to 12 . Eighty students from psychology classes at the University of Maryland, who had not been in Experiment 1, participated in order to gain extra course credit. Two additional subjects were tested, but their data were eliminated from the analyses for the same reason as in Experiment 1.

\section{Results}

The total percentage of correct recognition responses per trial for each of the four conditions is shown in Table 3. An analysis of variance revealed the same pattem of results as in Experiment 1 with the following significant effects: semantic vs. cheremic $[F(1,76)=49.53$, $\mathrm{p}<.001, \quad \mathrm{MSe}=288.77] ;$ trial $[\mathrm{F}(4,304)=933.39$, $\mathrm{p}<.001, \mathrm{MSe}=17.70]$; and semantic vs. cheremic by trial $[\mathrm{F}(4,304)=5.10, \mathrm{p}<.001$, MSe $=17.70]$. As in Experiment 1, the mean number of correctly recognized signs per trial was greater for the semantic list than for the cheremic list ( 43.81 vs. 31.85 ), and sign recognition improved between Trials 1 and 5 , as can be seen in Table 3. A Tukey HSD test showed that for each trial,

Table 3

Mean Percentage Correct Responses as a Function of Trial and Condition in Experiment 2

\begin{tabular}{lcccccc}
\hline & \multicolumn{6}{c}{ Trial } \\
\cline { 2 - 6 } \multicolumn{1}{c}{ Condition } & 1 & 2 & 3 & 4 & 5 & Mean \\
\hline Semantic Scrambled & 35.8 & 63.3 & 83.0 & 90.9 & 95.5 & 73.7 \\
Semantic Grouped & 35.4 & 64.8 & 81.2 & 88.1 & 92.0 & 72.3 \\
Cheremic Scrambled & 16.3 & 36.6 & 55.8 & 67.0 & 77.4 & 50.6 \\
Cheremic Grouped & 19.3 & 44.6 & 62.3 & 71.5 & 79.9 & 55.5 \\
Mean & 26.7 & 52.3 & 70.6 & 79.4 & 86.2 & \\
\hline
\end{tabular}

Table 4

Mean Number of Substitution Errors in Experiment 2 for Each Condition for All Trials Combined

\begin{tabular}{clccr}
\hline & & \multicolumn{2}{c}{ Presentation Order } & \\
\cline { 3 - 4 } List & Error & Grouped & Scrambled & Total \\
\hline \multirow{2}{*}{ Semantic } & Inside & 4.70 & 5.15 & 9.85 \\
& Outside & 13.55 & 17.95 & 31.50 \\
Cheremic & Inside & 12.65 & 16.40 & 29.05 \\
& Outside & 12.15 & 15.95 & 28.10 \\
\multirow{2}{*}{ Combined } & Inside & 17.35 & 21.55 & 38.90 \\
& Outside & 25.70 & 33.90 & 59.60 \\
\hline
\end{tabular}

the signs on the semantic list were recognized significantly better than on the cheremic list $(\mathrm{p}<.01)$. As in Experiment 1, the interaction between semantic vs. cheremic and trial seems to be due to a ceiling effect for the semantic list, even though the lists were twice as long as in Experiment 1.

For the overlapping signs, the percentage of correct responses showed a pattern of results similar to the overall results.

The mean number of substitution errors (inside set vs. outside set) are shown in Table 4. An analysis of variance performed on the logarithmic transformed substitution errors $\left[F_{\max }(8,20)=3.53, p>.05\right]$ revealed significant effects for the following: semantic vs. cheremic $[F(1,76)=18.56, p<.001, \quad M S e=.10]$; grouped vs. scrambled $[F(1,76)=7.54, \mathrm{p}<.01, \mathrm{MSe}=$ $.10]$; inside vs. outside $[\mathrm{F}(1,76)=48.60, \mathrm{p}<.001$, $\mathrm{MSe}=.04]$; semantic vs. cheremic by inside vs. outside $[\mathrm{F}(1,76)=63.16, \mathrm{p}<.001, \mathrm{MSe}=.04]$. More substitution errors were made for the cheremic list than for the semantic list (57.15 vs. 41.35 ). Scrambled presentation order resulted in more substitution errors than grouped presentation order (55.45 vs. 43.05). More outside-set errors were made than inside-set errors (59.50 vs. 38.90 ). (This result would be expected by chance, because the probability of an ouside error was much greater than the probability of an inside error, for example, $55: 4$ for groups of Size 5 and $51: 8$ for the sets of Size 9 , assuming only responses from the list were possible). A Tukey HSD test was performed on the differences between the means for the Semantic vs. Cheremic by Inside vs. Outside interaction. This test revealed that for the semantic list, more outside errors were made than inside errors $(31.50$ vs. $9.85, p<.01)$, whereas for the cheremic list, there was no difference between the number of inside and outside errors. There was more confusion between the signs within a set on the cheremic list (29.05) than on the semantic list (9.85).

In summary, the main results of Experiment 2 replicated the findings of Experiment 1 . The signs were easier to recognize in a list of semantically similar signs than in a list of cheremically similar signs. The order of presentation (grouped vs. scrambled) did not affect the number of signs correctly recognized. This pattern of results was 
obtained for the overlapping signs, as well as for the lists as a whole. More substitution errors, particularly from inside the set, were made on the cheremic list than on the semantic list. In Experiment 2, scrambled presentation resulted in more substitution errors than did grouped presentation.

Thus, the results of Experiments 1 and 2 suggest that it is more difficult to leam signs when other signs in the list are cheremically similar than when the other signs are semantically similar. The error data provides further evidence that subjects confused similar looking signs more often than those that were similar in meaning. However, it could be argued that the nonoverlapping signs (i.e., those that did not appear on both tapes) were more difficult on the cheremic list than on the semantic list, which could account for the obtained differences. To determine whether there were differences in the perceived difficulty of the signs on the cheremic and semantic lists used in Experiment 2, difficulty ratings of the individual signs were obtained from sign-naive subjects.

Ratings. Twenty additional sign-naive subjects rated the signs on a scale from 1 (easy) to 7 (hard) for how difficult each individual sign would be for them to recognize. Half of the subjects judged the signs on the cheremic list first, followed by those on the semantic list, and the other half judged the lists in reverse order. Before making the ratings, the subjects viewed a study trial (Trial 2 from the scrambled condition) to become familiar with the signs, and then the ratings were made (using test Trial 2). An analysis of variance was performed with list type (semantic vs. cheremic) and presentation order of lists (a counterbalancing factor) as factors. There were no significant differences in the mean difficulty rating of the signs on the semantic and cheremic lists (3.97 vs. 4.06). This was true despite the fact that the recognition performance during learning (the number of times a sign was correctly recognized in Trial 2 of Experiment 2) and the mean difficulty rating for the individual signs were correlated $(\mathrm{r}(92)=.57, \mathrm{p}<.001)$. Also, the intra- and intergroup reliability of the ratings was high. Based on the mean ratings of the overlapping signs (i.e., those signs that occurred on both the cheremic and semantic lists), the intragroup reliability (the agreement in the ratings of the cheremic and semantic lists) was $r(24)=.98$, $p<.001$. The intergroup reliability (the agreement between the mean ratings made by the subjects who saw the lists in the order semantic vs. cheremic with those who saw the lists in the opposite order) for all signs was $\mathrm{r}(92)=.84, \mathrm{p}<.001$. Thus, the raters were able to reliably and meaningfully rate the signs on difficulty.

\section{DISCUSSION}

The results of the present studies demonstrated that it is easier for sign-naive subjects to learn to recognize signs in the context of semantically similar signs than in the context of cheremically (formally) similar signs. Whether the presentation of the signs involved similar signs grouped together or not did not affect overall recognition performance.

The fact that the cheremically related signs were more difficult to recognize supports the expectations based on differentiation. Subjects had to learn the unique characteristics of the manual signs. For the cheremically similar signs, there were fewer unique physical characteristics than for the semantically similar signs. In addition, the subjects had a schema for dealing with semantic similarity, whereas they did not for cheremic similarity. Thus, it should have been, and was, more difficult to differentiate, encode, and recognize the cheremically similar signs than the semantically similar signs.

There are three ways that the present findings show that differences in acquisition of the semantic and cheremic lists are the result of the relationship between the signs, rather than the result of differences in the learnability in the individual signs on the lists. First, the same effect was found in both Experiments 1 and 2, which used different lists of signs (i.e., the semantic vs. cheremic effect is not specific to a given set of signs). Second, in both Experiments 1 and 2, the same pattern of results was obtained for the overlapping signs as for the lists as a whole, showing that learnability of the identical signs depended on the context (semantic or cheremic) in which they were presented. Third, the difficulty ratings of the signs of Experiment 2 showed that there were no differences in perceived difficulty of the individual signs on the semantic and cheremic lists. This result occurred even though the difficulty ratings showed high intra- and intergroup reliability, and were correlated with the learnability of the signs.

The interpretation in terms of differentiation is also supported by the error data. In general, in both experiments more substitution errors were made when the signs were cheremically similar than when they were semantically similar. More important, as expected on the basis of differentiation, signs within a set of cheremically similar signs were confused with each other to a greater extent than those within a set of semantically similar signs. The effect of semantic and cheremic context on outside errors was different in the two experiments. In Experiment 1, there were more outside errors in the cheremic condition than in the semantic condition, but in Experiment 2, there was no difference. This discrepancy in results is probably due to the increased number of overlapping signs in Experiment 2, which resulted in some cheremically similar signs on the semantic list and vice versa. As a result, some of the errors that were scored as outside errors on the semantic list in Experiment 2 were actually cheremically similar.

The results showing confusion of cheremically similar signs extend the results of Siple, Fisher, and Bellugi (1977). They found that in a recognition task, sign-naive 
subjects who were not informed of the sign meanings confused cheremically similar signs more often than cheremically dissimilar signs. The results of the present experiments show that information about sign meanings does not eliminate the confusion among cheremically similar signs.

Whether the similar signs were presented grouped together or were separated by other dissimilar signs (i.e., grouped or scrambled presentation order) did not affect the number of signs that were correctly recognized but did affect the type of substitution errors made. In Experiment 1 , scrambled presentation resulted in more inside-set errors than outside-set errors. In Experiment 2, scrambled presentation resulted in more inside- and outside-set substitition errors than did grouped presentation. The differences in pattern of substitution errors in the two experiments are probably attributable to different list lengths. The longer lists in Experiment 2 resulted in relatively more outside errors for both grouped and scrambled presentation than in Experiment 1, as would be expected on the basis of chance, since more outside errors were possible in Experiment 2.

One finding that was somewhat surprising in both experiments, and particularly in Experiment 2, was the rapid rate of acquisition, as indicated by the ceiling effect in the semantic condition. Previous studies (c.g., Gagné, 1950; Rothkopf, 1958, Runquist \& Runquist, 1978) have used shorter lists with no indication of such rapid learning. The iconic or representational property of manual signs (i.e., the fact that signs often resemble or suggest their referent) perhaps makes sign language easier to learn than arbitrary symbols and spoken languages (cf. Brown, Note 1). For example, the sign for "egg" looks similar to someone breaking an egg and the sign for "milk" looks like someone milking a cow. Iconic properties probably serve as a mnemonic device to aid in sign recognition for hearing adults.

The results of these experiments suggest that the learning of signs by hearing adults might progress faster when semantically similar signs are presented together than when cheremically similar signs are presented together. Thus, sign language textbooks that present semantically similar signs together (e.g., Conversational Sign Language II: An Intermediate-Advanced Manual by W. J. Madsen, 1972, or The Joy of Signing by L. Riekehof, 1978) should be used in order to facilitate sign recognition in hearing adults rather than those textbooks that present cheremically similar signs together. The conclusion that vocabulary learning might progress faster when semantically similar items are presented together than when formally similar items are presented together should apply to learning other second languages as well.

The present results must be interpreted cautiously for three reasons. First, it is possible that only for completely naive learners are cheremically similar signs more difficult than semantically similar signs. For example. despite lower recognition scores, subjects in the cheremic condition in the present studies may have learned more about the important distinctions between signs than subjects in the semantic condition. This could make it easier for subjects in the cheremic condition to learn a new list of signs. Although research is needed to determine the effects of prior experience on subsequent sign learning, the results of one recent study suggest that prior experience with sign language may change and reduce, but not eliminate, the difficulties in learning cheremically similar signs (Siple, Caccamise, \& Brewer, 1982). Second, no conclusion can be drawn concerning how acquisition would progress when there is no similarity in items compared with when there is semantic or cheremic similarity. A "no similarity" condition was not included in the present experiments because it seems that all sign textbooks (except dictionaries) are organized on the basis of cheremic or semantic similarity or a combination of those two dimensions. Third, only recognition, a receptive skill, was studied in the present experiments; therefore, no conclusions can be made about an equally important skill, the production of signs. Further research on the acquisition of manual signs by hearing adults is needed to address these three issues.

\section{REFERENCE NOTE}

1. Brown, $\mathbf{R}$. Why are signed languages easier to learn than spoken languages? Paper presented at the National Symposium of Sign Language Research \& Teaching, Chicago, May 1977.

\section{REFERENCES}

Anderson, J. R., \& Bower, G. H. Human associative memory. Washington, D.C: Winston, 1973.

BADDELEY, A. D. Effects of acoustic and semantic similarity on short-term paired-associate learning. British Journal of Psychology, 1970, 61, 335-343.

Battig, W. F. Paired-associate learning. In T. R. Dixon \& D. L. Horton (Eds.), Verbal learning and general behavior theory. Englewood Cliffs, N.J: Prentice-Hall, 1968.

GAGNÉ, R. M. The effect of sequence of presentation of similar items on the learning of paired associates. Journal of Experimental Psychology, 1950, 40, 61-73.

GiBson, E. J. A systematic application of the concepts of generalization and differentiation to verbal learning. Psychological Review, 1940, 47, 196-229.

Goss, A. E., \& NoDINE, C. F. Paired-associates learning: The role of meaningfulness, similarity, and familiarization. New York: Academic Press, 1965.

Horowitz, L. M. Associative matching and intralist similarity. Psychological Reports, 1962, 10, 751-757.

KEISTER, R. S. The effects of blocked versus randomized presentation orders on organization in paired-associate learning. Journal of Verbal Learning and Verbal Behavior, 1972, 11, 293-302.

KINTsCH, W. Memory and cognition. New York: Wiley, 1977.

Kućera, H., \& Francis, W. N. Computational analysis of present-day American English. Providence, R.I: Brown University Press, 1967.

MADSEN, W. J. Conversational sign language II: An intermediateadvanced manual. Washington, D.C: Gallaudet College, 1972.

Nelson, D. L., \& Rowe, F. A. Information theory and stimulus encoding in paired-associate acquisition. Journal of Experimental Psychology, 1969, 79, 342-346. 
O'Rourke, T. J. A basic course in manual communication (Rev. ed.). Silver Spring, Md: National Association of the Deaf, 1973.

Postman, L. The effects of language habits on the acquisition and retention of verbal associations. Journal of Experimental Psychology, 1962, 64, 7-19.

Riекеноғ, L. The joy of signing. Springfield, Mo: Gospel Publishing House, 1978.

Rotherg, I. C., \& Woolman, M. Verbal paired-associate learning as a function of grouping similar stimuli or responses. Journal of Experimental Psychology, 1963, 65, 47-51.

Rотнкорғ, E. Z. Stimulus similarity and sequence of stimulus presentation in paired associate learning. Journal of Experimental Psychology, 1958, 56, 114-122.

RUNquist, W. N. Functions relating intralist stimulus similarity to acquisition performance with a variety of materials. Journal of Verbal Learning and Verbal Behavior, 1968, 7, 549-553.

Runquist, W. N., \& Runquist, P. A. Interference reduction with conceptually similar paired-associates. Journal of Experimental Psychology: Human Learning and Memory, 1978, 4, 370-381.

Siple, P., Caccamise, F., \& Brewer, L. Signs as pictures and signs as words: Effect of language knowledge on memory for new vocabulary. Journal of Experimental Psychology: Learning, Memory and Cognition, 1982, 8, 619-625.

Siple, P., Fischer, S. D., \& Belluai, U. Memory for nonsemantic attributes of American Sign Language signs and English words. Journal of Verbal Learning and Verbal Behavior, 1977, 16, 561-574.
STE IN, B. S. Depth of processing reexamined: The effects of the precision of encoding and testing appropriateness. Journal of Verbal Learning and Verbal Behavior, 1978, 17, 165-174.

Stоков, W. C., JR. Sign language structure: An outline of the visual communication system of the American deaf. Studies in linguistics, occasional papers. Buffalo, N.Y: University of Buffalo Press, 1960.

Stokoe, W. C., Jr., Casterline, D., \& Croneberg, C. $A$ dictionary of American Sign Language. Washington, D.C: Gallaudet College, 1965.

UNDE RWOOD, B. J. Studies of distributed practice: IX. Learning and retention of paired adjectives as a function of intralist similarity. Journal of Experimental Psychology, 1953, 45, 143-149. (a)

UNDE RWOOD, B. J. Studies of distributed practice: VIII. Learning and retention of paired nonsense syllables as a function of intralist similarity. Journal of Experimental Psychology, 1953, 45, 133-142. (b)

\section{NOTE}

1. Although other criteria of cheremic similarity could have been employed in the selection of signs, their use would not be expected to affect the outcome of this study.

(Received for publication July 15, 1981; revision accepted April 28, 1982.) 